\title{
NEW DECIDABLE FIELDS OF ALGEBRAIC NUMBERS
}

\section{VAN DEN DRIES}

\begin{abstract}
A formally real field of algebraic numbers is constructed which has decidable elementary theory and does not have a real closed or $p$-adically closed subfield.
\end{abstract}

Introduction. In his list of problems [7], A. Robinson remarked (p. 501, loc. cit.): "I do not know of any proper subfield of the field of algebraic numbers, other than the fields of algebraic real or $p$-adic numbers, that has been shown to be decidable". Taken literally, this remark is rather strange, because the well-known results of Ax-Kochen-Eršov of 1964-1965 provide several decidable fields of algebraic numbers other than the fields mentioned by Robinson. But each of these is henselian with respect to a certain nontrivial valuation, so has a $p$-adically closed subfield for some prime $p$. (See [3] for the notion of $p$-adically closed field. A field of algebraic numbers is $p$-adically closed iff it is isomorphic with the field of algebraic $p$-adic numbers, similarly as a field of algebraic numbers is real closed iff it is isomorphic with the field of real algebraic numbers.)

It is also easy to see that a field extension of finite degree over a decidable field of algebraic numbers is a decidable field. But applying this result to one of the fields indicated above gives again fields with a $p$-adically closed or real closed subfield.

So probably Robinson wanted a decidable field of algebraic numbers which has no $p$-adically closed or real closed subfield. In $\$ 2$ we will construct such fields.

I am indebted to Jan Denef for calling my attention to the question answered in this paper.

1. Preliminaries. In this and the next section, $n$ is a fixed integer larger than 1. We define $O F_{n}$ as the 1st order theory whose models are the structures $\left(K, P_{1}, \ldots, P_{n}\right)$ with $\left(K, P_{i}\right)$ an ordered field, i.e. $K$ is a field and $P_{i}+P_{i} \subset$. $P_{i}, P_{i} \cdot P_{i} \subset P_{i}, P_{i} \cap P_{i}=\{0\}, P_{i} \cup\left(-P_{i}\right)=K(1<i<n)$. The language of $O F_{n}$ is $\left\{0,1,+, \cdot,-, \underline{P}_{1}, \ldots, P_{n}\right\}$, where $0,1,+, \cdot,-$ are the usual ring operation symbols and $\underline{P}_{1}, \ldots, \underline{P}_{n}$ are unary predicate symbols. The models of $O F_{n}$ are also called $n$-ordered fields.

Let us make a list of facts which we will need.

Received by the editors September 27, 1978.

AMS (MOS) subject classifications (1970). Primary 02H15, 02G05.

(C) 1979 American Mathematical Society 0002-9939/79/0000-0516/\$02.50 
Fact 1 (from [1, p. 54]; see also [5] for the notion of 'model companion'). $O F_{n}$ has a model companion $\overline{O F}_{n}$. The models of $\overline{O F}_{n}$ are those n-ordered fields $\left(K, P_{1}, \ldots, P_{n}\right)$ which satisfy:

(a) $P_{i}$ and $P_{j}$ induce different (interval) topologies on $K$, for all $i, j$ with $i \neq j$.

( $\beta$ ) For each irreducible $f(X, Y) \in K[X, Y]$, monic in $Y$, and each $a \in K$ such that $f(a, Y)$ changes sign on $K$ with respect to each of the orderings $P_{i}$, there exists $(c, d) \in K \times K$ with $f(c, d)=0$.

(In the formulation of [1, p. 54], $f(X, Y)$ in $(\beta)$ was not restricted to be monic in $Y$, but the usual 'linear transformation of variables' argument easily shows that we need only consider $f(X, Y)$ which are monic in $Y$.)

$\overline{O F}_{n}$ is even a decidable theory (cf. $[1$, p. 74]), but I do not see how this can be used to obtain a decidable model of $\overline{O F}_{n}$ which is algebraic over $\mathbf{Q}$. In $\$ 2$ we shall construct just such a model.

Fact 2. Suppose $K$ is an algebraic number field, $P_{1}, \ldots, P_{n}$ are different orderings on $K, f(X, Y) \in K[X, Y]$ is monic in $Y$ and irreducible, and $a \in K$ such that $f(a, Y)$ changes sign on $K$ w.r.t. each of the orderings $P_{i}$ on $K$. Then there is $a b \in K$ such that $f(b, Y)$ still changes sign on $K$ w.r.t. each $P_{i}$, and $f(b, Y) \in K[Y]$ is irreducible.

Because an algebraic number field is Hilbertian (cf. [4, Chapter 8]), and its different orderings induce different interval topologies, this fact follows from: if $\tau_{1}, \ldots, \tau_{n}$ are different nondiscrete $V$-topologies on a Hilbertian field $K$ and for each $i \in\{1, \ldots, n\} U_{i}$ is a nonempty $\tau_{i}$-open subset of $K$, while $H$ is a Hilbert set over $K$, then $U_{1} \cap \cdots \cap U_{n} \cap H \neq \varnothing$ (cf. [1, p. 62]).

Fact 3. There is an algorithm which, given $f(Y) \in \mathbf{Q}[Y] \backslash \mathbf{Q}$, decides whether $f(Y)$ is irreducible in $\mathbf{Q} Y \mathbf{Y}$. (In [8, p. 79] such an algorithm is given for $\mathbf{Z}[Y]$, and by Gauss' lemma we get one for $\mathbf{Q}[Y]$.)

Let $\tilde{\mathbf{Q}}$ be in the following a fixed algebraic closure of $\mathbf{Q}$. An algebraic number field is then any subfield $K$ of $\tilde{\mathbf{Q}}$ with $[K: \mathbf{Q}]<\infty$. We also fix a 1-1 map of $\tilde{\mathbf{Q}}$ onto a recursive subset of $\omega=\{0,1,2, \ldots\}$, such that addition and multiplication on $\mathbf{Q}$ correspond under this map with recursive functions. Let us call the image of $a \in \tilde{\mathbf{Q}}$ under this map the index of $a$. The existence of such an indexing is proved by Rabin in [6].

The phrase 'given $a \in \tilde{\mathbf{Q}}$ ' will simply mean: 'given the index of an element $a$ of $\tilde{\mathbf{Q}}$ '. Similarly a polynomial in $\tilde{\mathbf{Q}}\left[X_{1}, \ldots, X_{n}\right]$ is given if its degree $d$ is given and the vector of the coefficients of its monomials up to degree $d$ is given.

An index of an algebraic number field $K$ is the index of a generator $K$ over $\mathbf{Q}$, i.e. of an $a \in \tilde{\mathbf{Q}}$ with $K=\mathbf{Q}(a)$. 'Given an algebraic number field' will mean: 'given an index of an algebraic number field'.

Fact 4. There are algorithms (I), (II), (III), (IV), (V) such that:

(1) given $a \in \tilde{\mathbf{Q}}$, (I) determines the minimum polynomial of $a$ over $\mathbf{Q}$;

(2) given $a \in \tilde{\mathbf{Q}}$, (II) determines whether $a \in \mathbf{Q}$ holds; 
(3) given $a, b \in \tilde{\mathbf{Q}}$, (III) determines $c \in \tilde{\mathbf{Q}}$ with $\mathbf{Q}(a, b)=\mathbf{Q}(c)$;

(4) given $a, b \in \tilde{\mathbf{Q}}$, (IV) determines whether $\mathbf{Q}(a)=\mathbf{Q}(b)$;

(5) given an algebraic number field $K$ and $f \in K[Y] \backslash K$, (V) decides whether $f$ is irreducible in $K[Y]$.

We obtain (I) from Fact 3, (II) by using (I) and looking at the degree of the minimum polynomial. Given $a, b \in \tilde{\mathbf{Q}}$, there is a $c \in \tilde{\mathbf{Q}}$ with $\mathbf{Q}(a, b)=\mathbf{Q}(c)$, hence such a $c$ will be found by trying all possibilities, so (III) exists. Computing the degrees of $\mathbf{Q}(a), \mathbf{Q}(b)$ and $\mathbf{Q}(a, b)$ over $\mathbf{Q}$ by using (I) and (III) and looking at whether they are equal, gives (IV). A similar argument gives (V).

Suppose now that $a \in \tilde{\mathbf{Q}}$ has minimum polynomial $f(X) \in \mathbf{Q}[X]$ and that $f(X)$ has precisely $r_{f}$ real roots and that $r_{1}, \ldots, r_{n}$ are integers with $1<r_{1}<$ $r_{f}, \ldots, 1<r_{n}<r_{f}$. Let $\alpha \in \omega$ be the index of $a$. Then $\left(\alpha, r_{1}, \ldots, r_{n}\right)$ is said to be an index of the $n$-ordered field $\left(\mathrm{Q}(a), P_{1}, \ldots, P_{n}\right)$, where for each $i=1, \ldots, n P_{i}$ is the unique ordering on $\mathbf{Q}(a)$ such that $a$ is the $r_{i}$ th root of $f(X)$ in the real closure of $\left(Q(a), P_{i}\right)$, these roots being numbered in increasing order. Using (IV) and Sturm's theorem, the following will be clear:

Fact 5. There is an algorithm which, given $\left(\alpha, r_{1}, \ldots, r_{n}\right) \in \omega^{n+1}$, decides whether it is an index of an $n$-ordered field $\mathcal{K}$, and if so, computes the unique index $\left(\beta, s_{1}, \ldots, s_{n}\right)$ of $\mathscr{K}$ with minimal $\beta$.

Let us call this index $\left(\beta, s_{1}, \ldots, s_{n}\right)$ the minimal index of $\mathcal{K}$. It will now be clear what the phrase 'given an $n$-ordered algebraic number field' means.

Finally we will use in $\$ 2$ a fixed recursive bijection $\pi: \omega \rightarrow \omega \times \omega$ such that the first coordinate of $\pi(m)$ is $<m$, for all $m \in \omega$.

2. Construction of the field. Let $\mathscr{F}=\left(F, P_{1}, \ldots, P_{n}\right)$ be any given $n$ ordered algebraic number field such that $P_{i} \neq P_{j}$ for $i \neq j$. We define $\mathcal{C}$ as the set of all $n$-ordered algebraic number fields $\mathcal{K}$ with $\mathcal{F} \subset \mathcal{K}$. We fix for each $\mathscr{K} \in \mathcal{C}$ an enumeration $\alpha_{\mathscr{K}}:\left(f_{j}, a_{j}\right)_{j \in \omega}$ of all pairs $(f, a)$ with $f \in$ $K[X, Y]$ monic and of positive degree in $Y$, and $a \in K(K=$ the underlying field of $\mathscr{K})$. We suppose uniform effectiveness: there should be an algorithm which, given $\mathscr{K} \in \mathcal{C}$ and $j \in \omega$, constructs the pair $\left(f_{j}, a_{j}\right)=\alpha_{\mathfrak{K}}(j)$.

Now we can construct an ascending sequence $\left(\mathcal{K}_{m}\right)_{m \in \omega}$ in $\mathcal{C}$ as follows

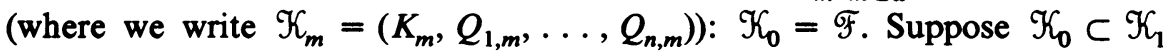
$\subset \cdots \subset \mathscr{K}_{m}$ have already been constructed. Let $\pi(m)=(i, j)$, so $i<m$. Then $\alpha_{\mathscr{F}_{1}}(j)$ is a pair $(f, a)$ with $f \in K_{i}[X, Y]$, monic and of positive degree in $Y$, and $a \in K_{i}$.

If $f(a, Y)$ does not change sign on $K_{m}$ with respect to one of the orderings $Q_{k, m}(1<k<n)$, then we put: $\mathcal{K}_{m+1}=\mathcal{K}_{m}$. Suppose $f(a, Y)$ changes sign on $K_{m}$ with respect to each of the orderings $Q_{k, m}$ on $K_{m}$. Then two cases can occur:

Case 1. $f(X, Y)$ is irreducible in $K_{m}[X, Y]$. In this case, there is by Fact 2 of 81 an element $c \in K_{m}$ such that $f(c, Y) \in K_{m}[Y]$ is still irreducible and still 
changes sign on $K_{m}$ with respect to each of the $n$ orderings $Q_{k, m}$ ( $k=$ $1, \ldots, n)$. Using (V) of Fact 4, $\$ 1$, and Sturm's theorem we will certainly find such a $c$ with the smallest possible index, and for this $c$ we compute the root $d \in \tilde{\mathbf{Q}}$ of $f(c, Y)$ with minimal index and define: $\mathcal{K}_{m+1}=\left(K_{m}(d)\right.$, $\left.Q_{1, m+1}, \ldots, Q_{n, m+1}\right)$, where $Q_{k, m+1}$ is the unique ordering on $K_{m}(d)$ extending $Q_{k, m}$, such that $d$ is the smallest root of $f(c, Y)$ in the real closure of $\left(K_{m}(d), Q_{k, m+1}\right)$.

Case 2. $f(X, Y)$ is reducible in $K_{m}[X, Y]$. If this is the case we will discover this by trying out decompositions of $f$. If we find one, we put $\mathcal{K}_{m+1}=\mathscr{K}_{m}$. By construction of the chain $\left(\mathcal{K}_{m}\right)_{m \in \omega}$ it is clear that the map $m \mapsto$ minimal index of $K_{m}$ is recursive.

We put $\mathscr{K}_{m}=\bigcup{ }_{m \in \omega} \mathcal{K}_{m}$, and write $\mathcal{K}_{\infty}=\left(K_{\infty}, Q_{1, \infty}, \ldots, Q_{n, \infty}\right)$.

Claim 1. $K_{\infty} \vDash \overline{O F}_{n}$. (See $\$ 1$, Fact 1.)

Proof. $Q_{1, \infty}, \ldots, Q_{n, \infty}$ are $n$ distinct orderings on $K_{\infty}$, because they extend the $n$ distinct orderings $P_{1}, \ldots, P_{n}$ on $\mathcal{K}_{0}$. As they are archimedean, they induce $n$ different interval topologies on $K_{\infty}$, so $(\alpha)$ of Fact 1 is satisfied. Suppose now that $f(X, Y) \in K_{\infty}[X, Y]$ is irreducible, monic in $Y$, and $f(a, Y)$ changes sign on $K_{\infty}$ with respect to each of the orderings $Q_{k, \infty}$, where $a \in K_{\infty}$. We have to show that $f(c, d)=0$ for some $(c, d) \in K_{\infty}^{2}$. Clearly there is $(i, j) \in \omega \times \omega$ with $\alpha_{\mathscr{F}_{t}}(j)=(f, a)$.

Let $m \in \omega$ be such that $\pi(m)=(i, j)$. Then by construction of the sequence $\left(\mathcal{K}_{m}\right)_{m \in \omega}$ we have: $K_{m+1} \vDash \exists c \exists d, f(c, d)=0$, so $K_{\infty} \vDash \exists c \exists d, f(c, d)=0$.

Claim 2. $\operatorname{Th}\left(\mathcal{K}_{\infty}\right)$ is decidable.

Proof. By model completeness of $\overline{O F}_{n}$ and Claim 1 we have that $\overline{O F}_{n} \cup$ $\operatorname{Diag}\left(\mathcal{K}_{\infty}\right)$ is a complete theory. But $\operatorname{Diag}\left(\mathcal{K}_{\infty}\right)=\cup\left\{\operatorname{Diag} \mathcal{K}_{m} \mid m \in \omega\right\}$, so $\operatorname{Diag}\left(\mathscr{K}_{\infty}\right)$ is recursively enumerable. Hence $\overline{O F}_{n} \cup \operatorname{Diag}\left(\mathscr{K}_{\infty}\right)$ is a complete theory with a recursively enumerable axiomatization. This implies in particular that there are two recursive functions, one enumerating $\operatorname{Th}\left(\mathscr{K}_{\infty}\right)$, the other enumerating $\left\{\sigma \mid \neg \sigma \in \operatorname{Th}\left(K_{\infty}\right)\right\}$ (= the complement of $\operatorname{Th}\left(\mathcal{K}_{\infty}\right)$ within the set of $O F_{n}$-sentences). Hence $\operatorname{Th}\left(\mathcal{K}_{\infty}\right)$ is decidable.

COROLlARY. $K_{\infty}$ is a decidable subfield of $\tilde{\mathbf{Q}}$ and does not have any real closed or p-adically closed subfield.

(Because $K_{\infty}$ is formally real, and $p$-adically closed fields are not formally real.)

REMARK. The above arguments simply constructivize the proof of Theorem (3.1) in Chapter II of [1].

\section{Behavior under finite extensions.}

LEMMA. Let the field $K$ be an algebraic extension of $\mathbf{Q}$. Then $K$ is an atomic model of $\operatorname{Th}(K)$. (The reader will see in the proof what this means.)

Proof. Let $\left(k_{1}, \ldots, k_{m}\right) \in K^{m}$. Clearly there is a formula $\theta\left(x_{1}, \ldots, x_{m}\right)$ in the language $\{+, \cdot,-, 0,1\}$ which is satisfied by only finitely many $m$-tuples 
in $K^{m}$, among which is $\left(k_{1}, \ldots, k_{m}\right)$. Take $M \geqslant 1$ minimal such that there is such a $\theta\left(x_{1}, \ldots, x_{m}\right)$ with $K \vDash\left(\exists^{! M}\left(x_{1}, \ldots, x_{m}\right) \theta\left(x_{1}, \ldots, x_{m}\right)\right) \wedge$ $\theta\left(k_{1}, \ldots, k_{m}\right) \cdot\left(\exists^{! M}\left(x_{1}, \ldots, x_{m}\right)\right.$ stands for: there are exactly $M m$-tuples such that.)

Let now $\phi\left(x_{1}, \ldots, x_{m}\right)$ be any formula with $K \vDash \phi\left(k_{1}, \ldots, k_{m}\right)$. We will show that $K \vDash \forall x_{1}, \ldots, \forall x_{m}\left(\theta\left(x_{1}, \ldots, x_{m}\right) \rightarrow \phi\left(x_{1}, \ldots, x_{m}\right)\right)$. If this were not the case, then put $\Psi\left(x_{1}, \ldots, x_{m}\right):=\theta\left(x_{1}, \ldots, x_{m}\right) \wedge \phi\left(x_{1}, \ldots, x_{m}\right)$, and we have: $K \vDash\left(\exists^{! M-i}\left(x_{1}, \ldots, x_{m}\right) \Psi\left(x_{1}, \ldots, x_{m}\right)\right) \wedge \Psi\left(k_{1}, \ldots, k_{m}\right)$ for some $i>1$, contradicting the minimality of $M$. So $\theta\left(x_{1}, \ldots, x_{m}\right)$ generates the type of $\left(k_{1}, \ldots, k_{m}\right)$ with respect to $\operatorname{Th}(K)$.

Corollary. Let the decidable field $K$ be an algebraic extension of $\mathbf{Q}$. Then each field extension $L$ of $K$ with $[L: K]<\infty$ is also a decidable field.

Proof. Let $L=K(a)$, and let $X^{m}+k_{1} X^{m-1}+\cdots+k_{m}$ be the minimum polynomial of $a$ over $K$. Let $\theta\left(x_{1}, \ldots, x_{m}\right)$ be a generator of the type realized by $\left(k_{1}, \ldots, k_{m}\right)$ in $K$ (which exists by the lemma). We consider now the 1st order theory $T_{(K, \theta)}$ whose models are the structures $\left(L^{\prime}, K^{\prime}, k_{1}^{\prime}, \ldots, k_{m}^{\prime}\right)$ such that $L^{\prime}$ is a field with subfield $K^{\prime} ; K^{\prime} \equiv K$ and $L^{\prime}=K^{\prime}\left(a^{\prime}\right)$ for some $a^{\prime}$ whose minimum polynomial over $K^{\prime}$ is $X^{m}+k_{1}^{\prime} X^{m-1}+\cdots+k_{m}^{\prime}$, and $K^{\prime}$ F $\theta\left(k_{1}^{\prime}, \ldots, k_{m}^{\prime}\right)$. Because $\operatorname{Th}(K)$ is decidable, $T_{(K, \theta)}$ has a recursive axiomatization. We claim that $T_{(K, \theta)}$ is a complete theory: it is easy to see that, given any sentence $\sigma$ in the language of $T_{(K, \theta)}$, one can construct a sentence $\bar{\sigma}$ in the language of rings such that for every model $\left(L^{\prime}, K^{\prime}, k_{1}^{\prime}, \ldots, k_{m}^{\prime}\right)$ of $T_{(K, \theta)}$ :

$$
\left(L^{\prime}, K^{\prime}, k_{1}^{\prime}, \ldots, k_{m}^{\prime}\right) \vDash \sigma \Leftrightarrow\left(K^{\prime}, k_{1}^{\prime}, \ldots, k_{m}^{\prime}\right) \vDash \bar{\sigma} .
$$

But for such a model we have: $\operatorname{Th}\left(K^{\prime}, k_{1}^{\prime}, \ldots, k_{m}^{\prime}\right)=\operatorname{Th}\left(K, k_{1}, \ldots, k_{m}\right)$. Combining this with the above equivalence we see that $T_{(K, \theta)}$ is complete. As it is also recursively axiomatizable, $T_{(K, \theta)}$ is decidable. Because $\left(L, K, k_{1}, \ldots, k_{m}\right) \vDash T_{(K, \theta)}, \operatorname{Th}(L)$ is decidable.

REMARK. I do not know whether the following converse holds. If $K, L$ are fields, $\mathbf{Q} \subset K \subset L, L \mid \mathbf{Q}$ is algebraic, $[L: K]<\infty$ and $L$ is a decidable field, is then $K$ a decidable field? If $\mathbf{Q}$ is replaced by a finite prime field, this is true by Eršov's classification of algebraic extensions of $\mathbf{F}_{p}$ with decidable theory (cf. [2]).

\section{REFERENCES}

1. L. van den Dries, Model theory of fields (Decidability, and bounds for polynomial ideals), Thesis, Utrecht, June, 1978.

2. Yu. L. Eršov, Fields with a solvable theory, Dokl. Akad. Nauk SSSR 174 (1967); English translation in Soviet Math 8 (1967), 575-576.

3. S. Kochen, Integer-valued rational functions over the p-adic numbers: a p-adic analogue of the theory of real fields, Proc. Sympos. Pure Math., vol. 12, Amer. Math. Soc., Providence, R.I., 1969, pp. 57-73.

4. S. Lang, Diophantine geometry, Interscience, New York, 1961. 
5. A. Macintyre, Model completeness, Handbook of Mathematical Logic, North-Holland, Amsterdam, 1977, pp. 139-180.

6. M. Rabin, Computable algebra: general theory and theory of computable fields, Trans. Amer. Math. Soc. 95 (1960), 341-360.

7. A. Robinson, Metamathematical problems, J. Symbolic Logic 38 (1973), 500-516.

8. B. L. van der Waerden, Moderne algebra. I, Springer-Verlag, Berlin and New York, 1930.

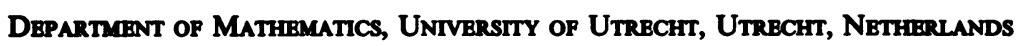

Current address: Department of Mathematics, Yale University, New Haven, Connecticut 06520 